\title{
Design methodology for shape transition inlets based on constant contraction of discrete streamtubes
}

Article in Proceedings of the Institution of Mechanical Engineers Part G Journal of Aerospace Engineering • November 2015

DOI: $10.1177 / 0954410015613112$

CITATIONS

0

4 authors, including:

\section{Yabin Xiao}

Chinese Academy of Sciences

2 PUBLICATIONS 0 CITATIONS

SEE PROFILE
READS

40

\section{Lianjie Yue}

Chinese Academy of Sciences, Institute of Me... 28 PUBLICATIONS 976 CITATIONS

SEE PROFILE 


\title{
Design methodology for shape transition inlets based on constant contraction of discrete streamtubes
}

Proc IMechE Part G:

$\int$ Aerospace Engineering

$0(0) \mathrm{I}-\mathrm{II}$

(C) IMechE 2015

Reprints and permissions:

sagepub.co.uk/journalsPermissions.nav

DOI: 10.1 |77/09544100156/31/2

uk.sagepub.com/jaero

(9SAGE

\author{
Yabin Xiao, Lianjie Yue, Shenghu Ma and Xinyu Chang
}

\begin{abstract}
In this paper we propose a design concept, i.e. constant contraction of discrete streamtubes, to develop a new design method for hypersonic inlets with shape transition, which enables the shape transition inlet to have high compression efficiency while keeping flow uniformity as much as possible. The method first divides the 3D flow field of the inlet into several groups of discrete streamtubes with constant contraction ratio. The axisymmetric flow within each stream tube is then optimized via computational fluid dynamics to have high compression efficiency, and these discrete streamtube groups are finally assembled together by matching their shock wave reflection positions to form a 3D inlet flow field with weak crossflow. This method can be used for the design of shape transition inlets with specified entrance/exit sections. To validate the present methodology, we design a rectangular-to-circular inlet with a design point of Ma 6 and a takeover point of $M a 4$, and examine numerically the flow structure and the inlet performances.
\end{abstract}

\section{Keywords \\ Hypersonic inlet, shape transition, inward turning, scramjet, design method}

Date received: 22 December 2014; accepted: 28 September 2015

\section{Introduction}

Scramjet, one of the most promising candidates for hypersonic air vehicles, mainly comprises inlet, isolator, combustor, and nozzle. For different components, the optimization target varies accordingly. For example, for the inlet-airframe integration, the capture shape for the inlet of each scramjet module is required to rectanglelike so that the modules may be mounted side-by-side. Generally, there is a preference for a circular combustor, which can withstand high pressure/thermal load and reduce unfavorable effects associated with corner flows. A desirable inlet feature for some scramjet applications is a shape transition from the rectangular-like capture to a circular throat so that the demands of different components can be met simultaneously.

Recently, the hypersonic inlets with shape transition have attracted wide interest. Series of design methods have been developed, including the following three categories.

1. Stream-tracing-based mathematical lofting method. A typical one is the hypersonic inlet with rectangular-to-elliptical shape transition (REST). ${ }^{1-3}$ Streamline tracing is first used to carve various inlets with the predefined rectangular-like capture shape or elliptical throat from the same compression field. A REST inlet is then generated by combining various stream traced inlet shapes with a mathematical lofting method developed by Barger $^{4}$ at the crosssections perpendicular to the entrance flow. This procedure enables smooth transition from specified capture to throat shape.

As is well known, the Busemann flow ${ }^{5,6}$ has a series of co-axial Mach cones with the same vertex, on each Mach cone the aerodynamic parameters keep unchanged. Referring to Busemann flows, Taylor and VanWie ${ }^{7}$ proposed a method to do the mathematical lofting along these Mach cones. The blending functions have great effects on the inlet performances. Among them the inverse tangent blending function was found to have the least total pressure loss and minimal drag. Nan et al. adopted the inlet design method described Taylor and Van $\mathrm{Wie}^{7}$ to generate an inlet with shape transition on the basis of an axisymmetric flow field with inverse tangent pressure rise. $^{8}$ The mathematical lofting method attempts to acquire a satisfactory inlet

State Key Laboratory of High Temperature Gas Dynamics, Institute of Mechanics, Chinese Academy of Sciences, China

\section{Corresponding author:}

Lianjie Yue, State Key Laboratory of High Temperature Gas Dynamics, Institute of Mechanics, Chinese Academy of Sciences, Number I5 Beisihuanxi Road, China.

Email: yuelj@imech.ac.cn 
flow field by coupling with streamline-tracing. However, the lofting of two families of streamlines does not involve any aerodynamic principles, and thus the resultant inlet flow cannot well maintain the characteristics of the parent flow field.

2. Section controllable internal waverider hypersonic inlet design ${ }^{9}$ using the theory of osculating axisymmetric flow. ${ }^{10}$ It uses a series of axisymmetric coaxial flows which have the same generatrix but different center body radii to realize smooth shape transition. This method endows the leading edge shock identical shock strength in the azimuthal direction. However, the reflected shock is left untreated, and thus the crossflow behind the reflected shock cannot be controlled. As a result, there are complicated 3D flow and shock trains in the isolator.

3. The direct optimization method. Sabean and Lewis ${ }^{11}$ applied the CFD-based direct optimization method to accomplish a rectangular to circular inlet design. However, its computational cost is very sensitive to the number of optimization parameters. ${ }^{12}$ The geometry profile of the inlet with shape transition is usually very complicated. If accurately portrayed, the computational cost will be too huge to be acceptable.

To address the abovementioned, the current paper proposes a new design method for shape transition inlets, in which smooth shape transition is accomplished by incorporating relevant aerodynamic principles. We first employ the idea of constant contraction to construct discrete streamtubes with high total pressure recovery coefficient, and then assemble them in a specific way to achieve fairly good flow uniformity. The nonaxisymmetric leading edge shock is designed to converge to a straight section of line so as to achieve wave-riding, corresponding to high compression efficiency in performance and uniformity in flow structure. The present method is hoped to provide a new thought for the design of shape transition inlets.

\section{Constant contraction and flow uniformity}

Necessary condition for the uniformity of flow field

Compression in the inlet is a thermodynamic process. Any two independent thermodynamic variables can

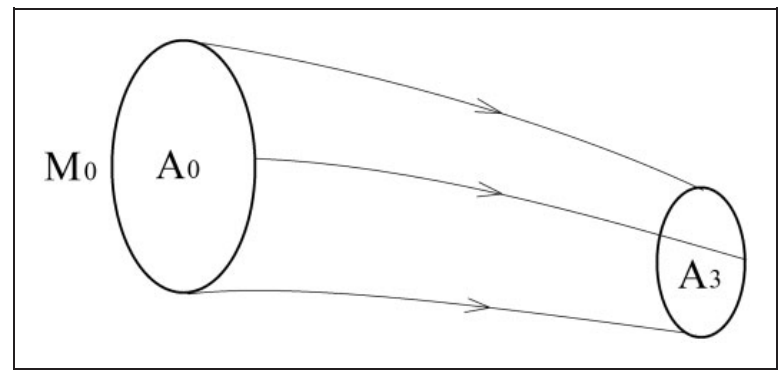

Figure I. Schematic of a streamtube in the inlet flow field. uniquely determine all other thermodynamic variables, and in turns determine, according to the energy conservation, the flow velocity and other aerodynamic parameters. Therefore, the necessary condition for uniform flow field is that any two thermodynamic variables are uniform there. Without loss of generality, we define them as pressure ratio $\pi_{30}$ and total pressure recovery coefficient $\sigma_{30}$.

For a streamtube in certain flow field, as shown in Figure 1, the entrance and exit areas are $A_{0}$ and $A_{3}$, respectively, and its contraction ratio is $C r_{03}=A_{0} / A_{3}$. Suppose the working medium is a perfect gas. Usually, the inlet flow can be considered as adiabatic, thus the total temperature is a constant. From the first law of thermodynamics, there is a relation between total pressure and entropy as follows

$$
\frac{\mathrm{d} P_{t}}{P_{t}}+\frac{\mathrm{d} s}{R}=0
$$

The entropy can be related to static temperature and static pressure as

$$
\frac{C_{p}}{R T} \mathrm{~d} T=\frac{\mathrm{d} P}{P}+\frac{\mathrm{d} s}{R}
$$

Combination of equations (1) and (2) yields

$$
\frac{\gamma}{\gamma-1} \frac{\mathrm{d} T}{T}=\frac{\mathrm{d} p}{p}-\frac{\mathrm{d} P_{t}}{P_{t}}
$$

From the equation of state and the mass conservation, we have

$$
\frac{\mathrm{d} A}{A}=\frac{1}{\gamma} \frac{\mathrm{d} p}{p}-\frac{\gamma-1}{\gamma} \frac{\mathrm{d} P_{t}}{P_{t}}+\frac{\mathrm{d} u}{u}
$$

Integrating along a streamtube, we get

$$
C r_{03}=\frac{A_{0}}{A_{3}}=\pi_{30^{\frac{1}{\gamma}}} \sigma_{30}-\frac{\gamma-1}{\gamma} \cdot \frac{u_{3}}{u_{0}}
$$

Based on the energy conservation, we can, for incoming Mach number of $M a_{0}$, derive a relation between geometric contraction ratio $\mathrm{Cr}_{03}$, pressure ratio $\pi_{30}$ and total pressure recovery coefficient $\sigma_{30}$ as follows

$$
C r_{03}=\pi_{30}{ }^{\frac{1}{\gamma}} \sigma_{30}-\frac{\gamma-1}{\gamma} \sqrt{1+\frac{2}{(\gamma-1) M_{0}^{2}}\left[1-\left(\frac{\pi_{30}}{\sigma_{30}}\right)^{\frac{\gamma-1}{\gamma}}\right]}
$$

Equation (6) shows that if the pressure ratios $\pi_{30}$ and total pressure recovery coefficients $\sigma_{30}$ of any two streamtubes are equal, their contraction ratios are identical too. The property that any streamtube in a flow field has the same contraction ratio is defined in this 
paper as "constant contraction." Hence, "constant contraction" is the necessary condition for the flow uniformity at the inlet exit. Certain classic flows such as Busemann flow ${ }^{13}$ do satisfy this condition. If an inlet flow is not of "constant contraction" type, some of the streamtubes would have larger contraction ratio as well as higher pressure ratio, temperature rise, and entropy rise. While for some others the situation is on the contrary. This is an important source for the nonuniformity of flow fields.

\section{Weakened version of the "constant contraction" condition}

The "constant contraction" condition is so strict that it can hardly be met in practical designs of hypersonic inward turning inlets because the similarity condition is difficult to hold in rotational flows. For shortening the compression surface and reducing the viscous loss, it is necessary to truncate the Busemann flow field or replace it by other similar compression flow field with limited length, as shown in Figure 2.,14,15 This kind of basic flow fields share a similar flow structure, including incident shock, isentropic compression, and reflected shock. For the internal conical flow, the incident shock TP inevitably intercepts the concurrent characteristic lines and turns gradually inward accordingly, resulting in a rotational flow downstream. The entropy rise across the shock grows with decreasing radius. Hence, there are entropy gradients between different streamlines in the isentropic compression region (TPC). This kind of inward turning axisymmetric flow fields containing incident axisymmetric shocks does not satisfy the "constant contraction" condition, and cannot develop into a strictly uniform flow.

For all this, it is still possible, under specific division, to collect certain streamtube groups with constant contraction ratio. As shown in Figure 3, for discrete sector-shaped streamtubes within an axisymmetric internal conical flow, $T_{i} T_{i+1} S_{i+1} S_{i}-C_{i} C_{i+1} S_{i+1}^{\prime} S_{i}^{\prime}(i=0$, $1, \ldots)$, their average flow parameters are equal because the aerodynamic parameters keep unchanged in the circumferential direction. Nevertheless, nonuniformity is allowed within each streamtube. The division of

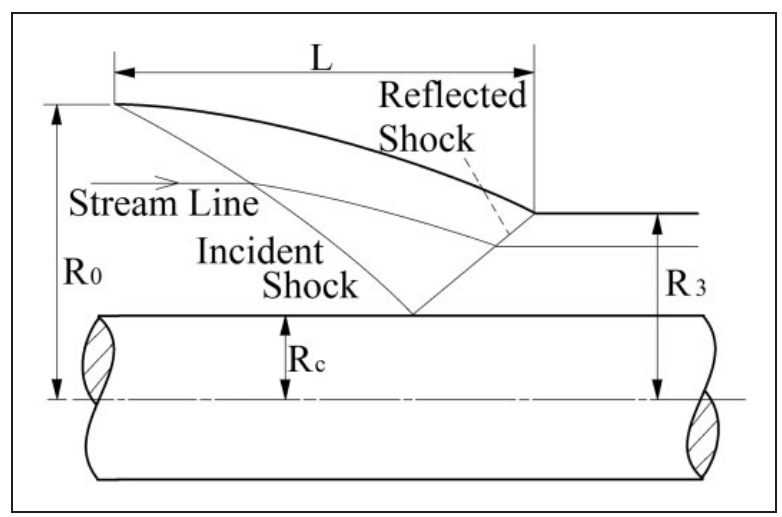

Figure 2. Schematic of shock waves in axisymmetric flow. streamtubes is not arbitrary, but is carried out in a specified manner instead (division along the radial direction, as shown in Figure 3). Therefore, it is a weakened version of the "constant contraction" condition proposed in section "Necessary condition for the uniformity of flow field", called as "constant contraction of discrete streamtubes".

In the design of hypersonic inlets with shape transition, we will employ specific division method to make the discrete streamtubes acquire, as much as possible, the property of weakened "constant contraction". According to equation (6), with fixed contraction area ratio of discrete streamtubes, we need just to improve the consistency of one of the averaged thermodynamic parameters, then the overall flow uniformity would be achieved automatically.

\section{Design methodology of shape transition inlets}

\section{Division of the 3D inlet flow field into discrete streamtubes with constant contraction ratio}

The entrance and exit profiles of the inlet with shape transition are usually specified in advance. The division of streamtubes is carried out in a plane perpendicular to the entrance flow. To facilitate the matching of adjacent streamtubes, we divide the flow field into several streamtubes, each of which is a sector part of a certain axisymmetric flow. Figure 2 shows an axisymmetrically contraction flow with a center cylinder, with its contraction ratio of

$$
C r=\frac{R_{0}{ }^{2}-R_{c}{ }^{2}}{R_{3}{ }^{2}-R_{c}{ }^{2}}
$$

Billig $^{13}$ introduced a parameter, called as radial deviation parameter (RDP), to describe an axisymmetric flow with a center cylinder

$$
R D P=\frac{R_{0}-R_{c}}{R_{0}}
$$

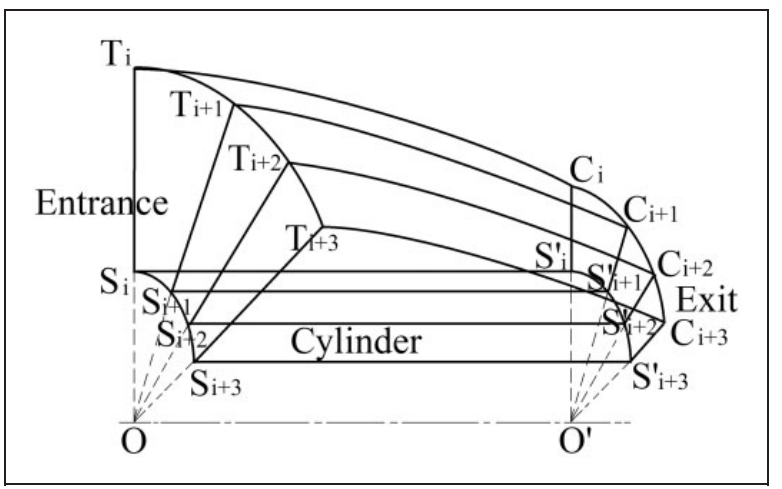

Figure 3. Division of discrete streamtubes with "weakened constant contraction" in axisymmetric flow fields. 
For inward turning axisymmetric flows, this parameter, which ranges in value from 0 to 1 , spans a continuous spectrum from two-dimensional planar $(\mathrm{RDP}=0)$ to maximum inward turning with center cylinder radius of $0(\mathrm{RDP}=1)$. We can make use of a series of discrete axisymmetric streamtubes with the same contraction ratio and continuously varying RDP to divide arbitrary entrance/exit sections.

A rectangular to circular inlet with right-left symmetry is taken as an example to illustrate the present method, as schematically shown in Figure 4. The quantity of the stream tubes can be arbitrary. In general, increase in the number of the stream tubes helps make the inlet surfaces smoother and more accurate, but inevitably consumes more time.

The division starts from the top of the symmetric plane, firstly the capture lines on the top and the side walls are divided into $N$ segments, and the division may be not equally spaced. $N+1$ straight lines are drawn through points $T_{0}, T_{1}, T_{2}, \ldots T_{N-1}, T_{N}$ to divide the near-rectangular capture area into $N$ parts. The intersection point between the $i$ th straight line and the projection of the inlet's circular exit is point $C_{i}$, and the intersection point between the ith straight line and the symmetric line is point $S_{i}$. In order to make the ratio of the capture area of each streamtube $T_{i} S_{i} S_{i+1} T_{i+1}$ to the outlet area $C_{i} S_{i} S_{i+1} C_{i+1}$ acquire the same contraction ratio as the overall contraction ratio of the inlet, we need to determine the position of point $S_{i}$ consecutively.

First, determine the position of $S_{0}$. Here we choose the center cylinder radius as zero, i.e. $\mathrm{RDP}=1$, and thus $S_{0}$ coincides with $S_{1}$. Based on specified $T_{1}$, point $S_{1}$ can be uniquely determined to get $\frac{A_{S_{1} T_{1} T_{0}}}{A_{S_{1} C_{1} C_{0}}}=C r_{03}$. Repeat the above procedure, for known $S_{i}, T_{i}, T_{i+1}$, we can successively determine $\mathrm{S}_{\mathrm{i}+1}$ to get $\frac{A_{T_{i} S_{S} S_{i+1} T_{i+1}}}{A_{C_{i} S_{i} S_{i+1} C_{i+1}}}=C r_{03}$.

Index $i$ runs from 0 to $N-1$, all stream tubes are a sector part of certain inward turning axisymmetric flow with a center cylinder. When the division proceeds to the lower half circle of the inlet exit, $\left|C_{N} S_{N}\right|$ decreases quickly. Because $\left|T_{N} S_{N}\right|$ must be larger than half of the entrance width, there will be a point $T_{N}$ on the side wall where $\left|T_{N} S_{N}\right| /$

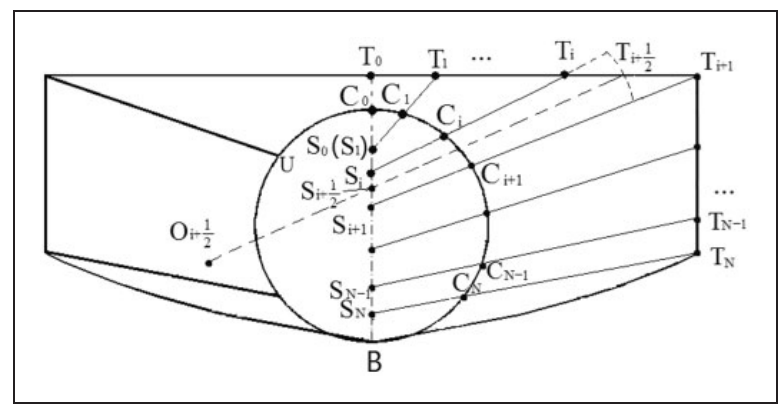

Figure 4. Schematic of the division of discrete streamtubes for a rectangular-to-circular inward turning inlet.
$\left|C_{N} S_{N}\right|=C r_{03}$, i.e. the flow is reduced to a $2 \mathrm{D}$ one with $\mathrm{RDP}=0$. Beyond this point there will not be any inward turning streamtubes, and the point of $T_{N}$ is taken as the terminal point in the division. Point $T_{N}$ is also the terminal point of the side wall, and $T_{N} S_{N}$ is the terminal line in side wall division. Curve $T_{N} B$ is projection of the cowl lip, which is not specified in advance, but is determined by $C_{N} B$ (the part of the circular outlet that is below $T_{N} S_{N}$ ) instead. It is generated by stream-tracing technique from the 2D compression flow field mentioned above.

When the division is sufficiently refined, every streamtubes obtained from the division can be viewed as a sector part of certain axisymmetric flow with the same contraction ratio and continuously varying RDP. For an arbitrary inlet capture shape, we can always find a way to divide the $3 \mathrm{D}$ flow field into a set of discrete stream tubes with constant contraction ratio.

\section{Design of the flow field inside discrete streamtubes}

On the basis of streamtube division, it is also necessary to make efficient design for each streamtube. A three-dimensional streamtube in Figure 4 is magnified in Figure 5, with $T_{i} S_{i} S_{i+1} T_{i+1}$ as the entrance and $C_{i} S_{i}^{\prime} S_{i+1}^{\prime} C_{i+1}$ as the exit. Its planar stream surface $S_{i} S_{i}^{\prime} C_{i} T_{i}$ and $S_{i+1} S_{i+1}^{\prime} C_{i+1} T_{i+1}$ are shared with adjacent streamtubes, while $T_{i} T_{i+1} C_{i+1} C_{i}$ is part of the inlet profile. When the division is sufficiently refined, we can take the axisymmetric flow on the streamtube central stream surface $T_{i+1 / 2} S_{i+1 / 2} S_{i+1 / 2}^{\prime} C_{i+1 / 2}$ to represent the flow in streamtube itself. Here, local axis is $O_{i+1 / 2} O_{i+1 / 2}^{\prime}$, local center cylinder radius is $\left|O_{i+1 / 2} S_{i+1 / 2}\right|$, and the contraction ratio is the same as that of the inlet. And thus the design of streamtube is reduced to that of an axisymmetric flow with $T_{i+1 / 2}$ $C_{i+1 / 2}$ as its generatrix.

Two factors need to be considered in the streamtube design. First, the compression with high efficiency is required. Second, all the designed streamtubes should have as close performances as possible, which not only guarantees the uniformity

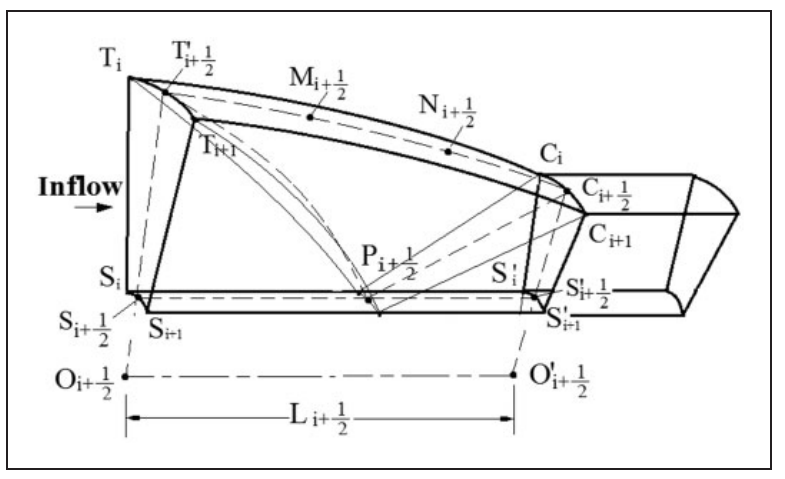

Figure 5. Three-dimensional structure of the axisymmetric streamtube. 
of the inlet flow field, but also endows the adjacent streamtubes approximately matched parameters so that the crossflux through $S_{i} S_{i}^{\prime} C_{i} T_{i}$ and $S_{i+1} S_{i+1}^{\prime}$ $C_{i+1} T_{i+1}$ are as low as possible, i.e. the desired streamtube flow can be achieved. Figure 5 depicts the shock pattern in the designed axisymmetric flow. The incident shock $T_{i+1 / 2} P_{i+1 / 2}$ intercepts the center cylinder at point $P_{i+1 / 2}$, and its reflected shock is $P_{i+1 / 2} C_{i+1 / 2}$ and the contraction section length is $L_{i+1 / 2}$. In order to get the best compression efficiency and make the reflected shock impinge on the shoulder (to weaken as far as possible the shock reflection in the isolator), we employ CFD based optimization method to design the axisymmetric profile $T_{i+1 / 2} M_{i+1 / 2} N_{i+1 / 2} C_{i+1 / 2}$. A strategy with total pressure recovery as the optimization target is proven to let the reflected shock impinge on the shoulder. The optimization depends to some extent on the length height ratio, i.e. $L_{i+1 / 2} / \mid T_{i+1 / 2}$ $S_{i+1 / 2} \mid$ in Figure 5. We found via numerical experiments that taking the same length height ratio can bring similar flow patterns and make compression ratios come close, which is beneficial to flow uniformity and streamtube matching. For simplicity, we take 5.0 as the common length height ratio for all streamtubes in present inlet design. Of course, adjusting the length height ratio to suit local condition can, with expense of computation cost, enhance the consistency of these discrete streamtubes.

In the present paper, profile $T_{i+1 / 2} M_{i+1 / 2} N_{i+1 / 2} C_{i+1 / 2}$ is expressed by a nonuniform rational B-spline (NURBS curve), in which the axial coordinates of $M_{i+1 / 2}$ and $N_{i+1 / 2}$ are set to $L_{i+1 / 2} / 3$ and $2 L_{i+1 / 2} / 3$. Optimization parameters are the vertical coordinates of $M_{i+1 / 2}$ and $N_{i+1 / 2}$, while the optimization target is the total pressure recovery which varies sharply with the optimization parameters. In the CFD optimization, the CFD-based optimization adopts MATLAB as the working environment, Gambit as the mesh generator, and FIUENT as the viscous CFD solver. The pattern search optimization algorithm in the MATLAB toolbox was employed, ${ }^{16-18}$ which does not need the calculation of gradient. The quadrilateral grids for these computations were generated using Gambit, and the mesh is refined near walls. In Fluent solver, AUSM scheme is adopted for flux splitting of the inviscid flux with second-order upwind scheme. The k- $\omega$ SST turbulence model is adopted to simulate the turbulent flow field. Mesh generation, CFD computation and data processing are automatically implemented by an objective function freely running relevant software in the optimization procedure through script file.

\section{Assembling and matching of discrete streamtubes}

Since all streamtubes have the same length height ratio and the same contraction ratio, they have similar flow structure and close aerodynamic performances. In addition, their heights $\left(R_{0}-R_{c}\right.$ in Figure 2$)$ and RDP are varied continuously and slightly. All these provide favorable conditions for the assembling and matching of streamtubes. We need only to manually control the streamwise positions of the reflection points of all incident shock waves (point $P_{i+1 / 2}$ in Figure 5), where the pressure varies most fierce. If points $P_{i+1 / 2}$ of all streamtubes are not matched, there will be a great crosswise pressure gradient, and in turn a strong crossflow. Hence, in assembling discrete streamtubes, the reflection points of all incident shock waves are specified with the same streamwise coordinate, whereby the reflection points of all incident shock wave will theoretically form a straight shock-reflection line vertical to both the incoming flow and the spanwise axis, denoted as $S_{0} B$ in Figure 4.

When the shock-reflection points are matched, the crosswise pressure gradient between the isentropic flows within adjacent streamtubes is determined by the difference of RDP and length height ratio. In the design shown in Figure 4, RDP varies slightly in region $i<N-1$, and thus the crosswise pressure gradient is small. In this sense, the assembled flow can be viewed as a generallized axisymmetric flow with weak crossflow. When the division comes to the terminal point on side wall, RDP will be quickly reduced to zero, and there is thus a certain crosswise pressure gradient between streamtube $N-1$ and streamtube $N$.

\section{Configuration design of a rectangular to circular inlet}

To validate the present method, a hypersonic inward turning inlet with shape transition is designed, and its

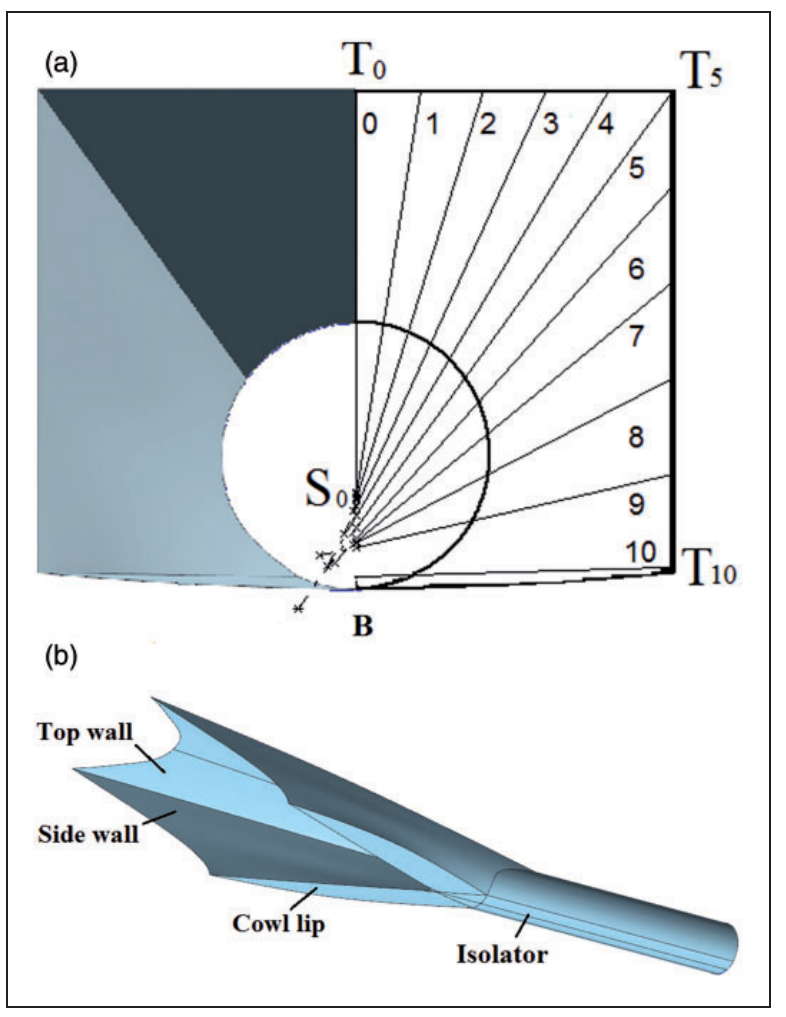

Figure 6. Schematic of the rectangular to circular inlet: (a) division of the discrete streamtubes; (b) configuration of the inlet. 
performances are examined at the design point of $M a 6$ and the takeover point of $M a 4$. The inlet entrance width is $190 \mathrm{~mm}$, and its height is $150 \mathrm{~mm}$. The diameter of the isolator is $80 \mathrm{~mm}$ and the inlet contraction ratio $\mathrm{Cr}_{03}$ is 5.6.

The inlet configuration is shown in Figure 6 . As can be seen in Figure 6(a), the top and side walls of the right half inlet is divided into 20 axisymmetric streamtubes with the same contraction ratio of 5.6. The projection of the boundary stream surfaces of some streamtubes are depicted with serial number marked.

The generated inlet configuration is shown in Figure 6(b). Its contraction section length is $813 \mathrm{~mm}$, and the isolator length is set to $400 \mathrm{~mm}$. Referring to Smart, ${ }^{1}$ we cut the V-shape cowl a little backward. In this way, the internal contraction ratio is reduced, and in turn, the range of operating Mach number is extended. Moreover, the leading edge shock is prevented from producing type IV interference with the bow shock of practical blunted cowl lip at design point. ${ }^{19}$ The backward cutting corresponds to an increase of $1.74^{\circ}$ in the sweep angle of the cowl, and the internal contraction ratio of the inlet is reduced to 1.67 accordingly. From the final simulation result, the leading edge shock did deviate a little from the cowl lip at the design point; however, we achieved the extension of operation range at a slight expense of $0.05 \%$ mass capture loss.

\section{Computation and analyses of the inlet flow field}

The flow structure and performances of the inlet are numerically examined at both the design and the takeover points by using FLUENT. Detailed numerical setup is the same as that introduced in section "Design of the flowfield inside discrete streamtubes". The computational domain is shown in Figure 7, in which only half of the flow field is simulated in view of

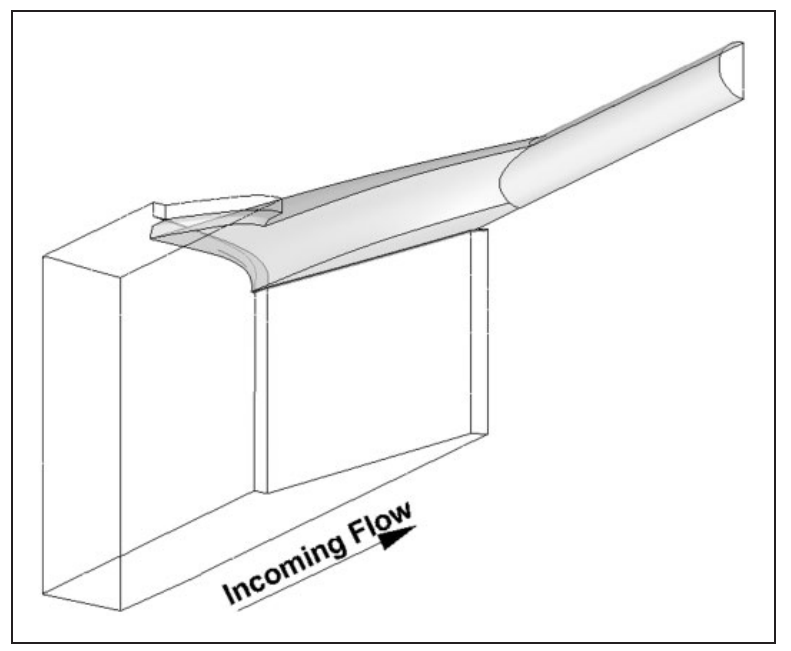

Figure 7. Schematic of the computational domain. the symmetry. Considering the inlet spillage, the computational domain is appropriately extended from the leading edge and the cowl. Far-field conditions are given at the upstream boundaries and pressure outlet condition is specified at the exits, which is essentially an extrapolation condition for supersonic flow. The computation mesh is a hybrid one generated by ICEM CFD. The height of the first boundary layer grids is $5 \mu \mathrm{m}$. The cells in the core region are of tetrahedron type, and the total number of cells amounts to 2.6 million. The residual tolerance for iteration convergence is set to $10^{-5}$. The mesh is found to offer sufficient calculation precision in the mesh independence test.

\section{Flow structure at the design point}

According to the original idea of design, nonaxisymmetric leading edge shock wave converge to a vertical shock-reflection line at $X=0$ (the axial coordinate of point $\mathrm{P}$ is defined as zero), as denoted by $\mathrm{SB}$ in Figure 6(a). Calculated Mach number contour and pressure ratio contour on the inlet symmetric plane are shown in Figure 8. This shock-reflection line can be seen from Figure 8(b), and it accomplishes basically the design target. Different from the design, the flow, after passing the free-standing reflected shock, continues to turn downward due to the spillage caused by the backward cutting of the cowl lip. This generates an additional reflected shock at the cowl which reflects again in the isolator and forms a train of weak oblique shock waves.

To describe detailed flow structure, we select and analyze the flow pattern on seven key slices within the inlet flow field, as shown in Figure 9. The distance between the leading edge and the designed shock reflection position is $D=563 \mathrm{~mm}$. Slices 1 to 7 are

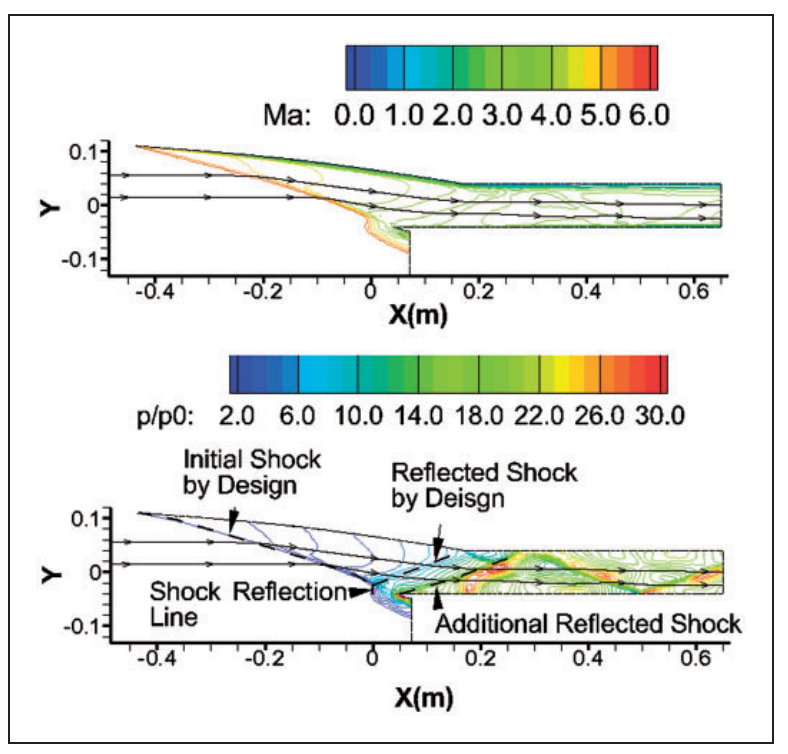

Figure 8. Contours of Mach number and pressure ratio on the symmetric plane at the design point. 
located with $\mathrm{X}$ coordinate of $-0.4 D,-0.2 D, 0,0.1 D$, $0.2 D, 0.72 D$, and $1.154 D$ to view the local flow patterns. Figure 10 shows numerical results at these key sections, contour of Mach number on the left side, while contour of pressure ratio on the right. It can be seen from Figure 10(a) and (b) that the leading edge shock is a nonaxisymmetric one, quite close to the cowl lip. The distance between them becomes increased in downstream region, resulting from the backward cutting of the cowl lip. If the cowl was not cut backward, the leading edge shock would fall on the edge of cowl lip and a perfect waveriding would be achieved. As can be seen from the pressure ratio contour shown in Figure 10(a) and (b), on the same slice the pressure ratio behind the leading edge shock is less than that near the compression surface. Since the near wall flow experiences an isentropic compression in addition to the leading edge shock compression, there occurs an additional pressure rise, as shown in Figure 8.

Figure 10(c) depicts the flow structure at the section of $X=0$. There is a shock in the lower part, and the contours of $M a 5$ and pressure ratio 2.6 indicates its position and configuration. Free-standing reflection shock of nonaxisymmetric leading edge shock arises. This shows that the present design leads successfully to the predetermined shock configuration, and the proposed method is successful. As shown in Figure 10(c) and (d), the pressure and Mach number are basically uniformly distributed in most regions upstream of the reflected shock.

According to the design, the leading shock should lead to a unique reflected shock behind the shock reflection line. However, as shown in Figure 8, due to the backward cutting of cowl lip, there occurs an additional reflected shock, leading to a pressure increase downstream. This reflected shock interacts with the side boundary layer to produce a region with thickened boundary layer, as shown in the left of Figure 10(e).

Flows at the exit and in the middle segment of isolator are shown in Figure 10(f) and (g), in which the sectional area of low-speed region is enlarged by viscous dissipation. The additional reflected shock can

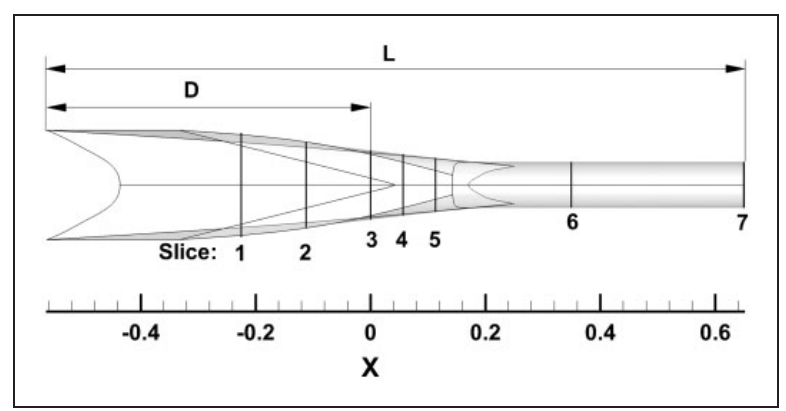

Figure 9. Key streamwise positions in the flow field. be observed to produce a weak oblique shock train, which would damage to some extent the flow uniformity and cause certain deviation of pressure distribution at the exit of the inlet. Essentially, this nonuniformity generated by reflected shocks is similar to that caused by reflected shocks in the isolator due to incomplete shock cancellation in $2 \mathrm{D}$ inlet. However, this kind of nonuniformity, unlike that resulting from complicated $3 \mathrm{D}$ flows, is generally acceptable. Furthermore, since the shock train is weak, the Mach number distribution in core flow is not much sensitive to the shock and remains quite uniform. In practical applications, air vehicles cannot strictly operate as designed and boundary layer effects are unavoidable, and thus weak reflected shocks in the isolator are acceptable.

According to the design, the flow field is composed of a series of discrete streamtubes with planar stream surfaces, where the crucial step is a proper matching between streamtubes to avoid strong crossflows. In order to validate the present method, we need to examine both the flow within and the flow crossing these typical stream surfaces.

We select slices 2, 4, 6, and 9 shown in Figure 6(a) as typical surfaces, and depict the corresponding Mach number contours in Figure 11. Abscissa X denotes the streamwise distance in $\mathrm{mm}$, and ordinate Y1 denotes the distance from points at the slice to the intersection line of the slice with the inlet symmetric plane. As can be seen from Figure 11(a) to (d), on typical slices, the abscissa of the intersection point of the leading edge shock with the symmetric plane $(Y 1=0)$ is quite close to zero, the reflected shocks impinge on the shoulder and no large scale separation is observed.. The flow fields are in accord with those predicted in the design, showing that the desired flow features are successfully reproduced.

To examine the crossflow across adjacent streamtubes, we define a nondimensionalized cross-stream intensity (shortened to CSI), i.e. the ratio of the crossflow velocity to the local velocity. CSI contours on the four streamwise slices are depicted in Figure 12. It can be seen that behind the leading edge shock, there are crossflows vertical to the side boundaries of discrete streamtubes. However, the circumferential pressure difference is not large (as shown in Figure 10(a) to (d)), and the crossflow intensity is mostly below $3 \%$, with maximum of $7 \%$ near shock or in the boundary layer. The crossflow in core region is not strong and deemed acceptable, in view of the complicated flow within shape transition inlets. It implies that the proposed method is feasible for inlet design.

\section{Flow structure at the takeover point}

Figure 13 presents the flow field on the inlet symmetric plane at the takeover point, where the angle of the leading edge shock increases, and spillage occurs upstream the cowl lip. The condition for 


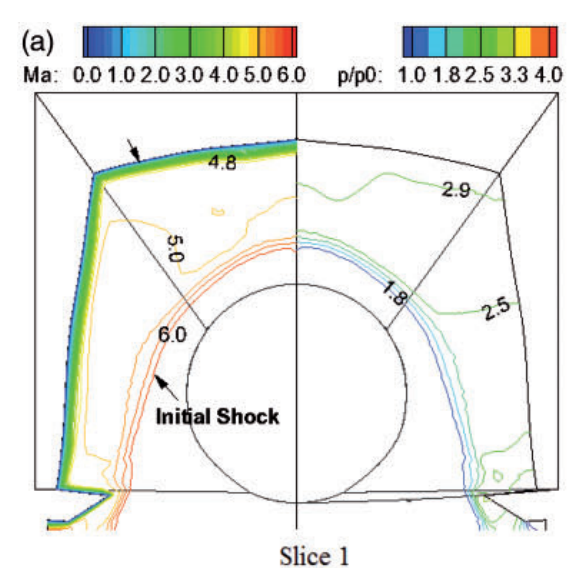

(c)

Ma: 0.0102 .030405 .060 p/p0: 1025355984

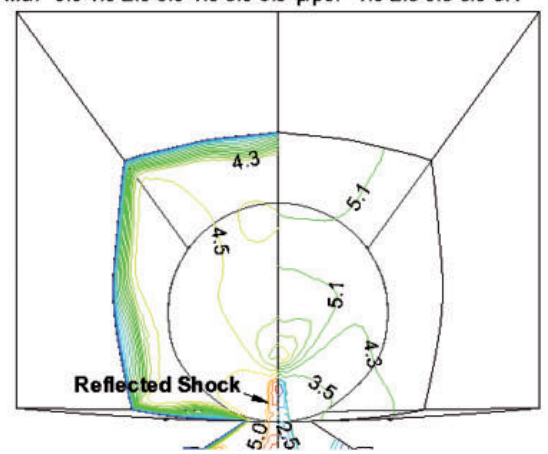

Slice 3

(e)

$\begin{array}{lllllll}\text { Ma: } 0.00 .81 .72 .53 .34 .25 .0 & \mathrm{p} / \mathrm{p} 0 \text { : } & 1.0 & 6.7 & 12.3 & 18.0\end{array}$

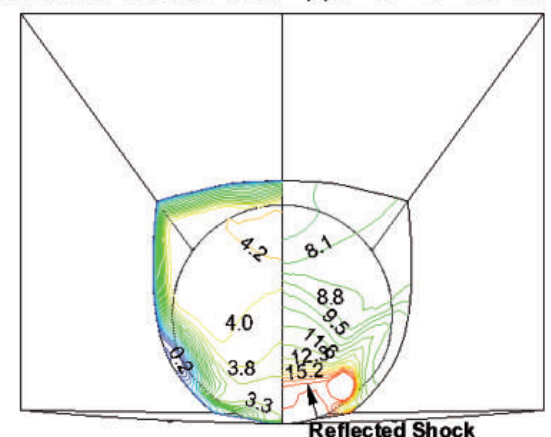

Slice 5

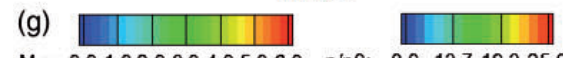

Ma: 0.01 .02 .03 .04 .05 .06 .0 p/p0: 8.013 .719 .325 .0

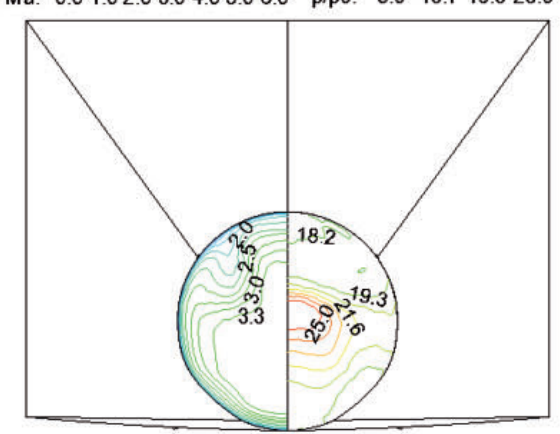

Slice 7

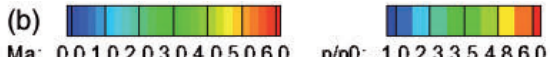

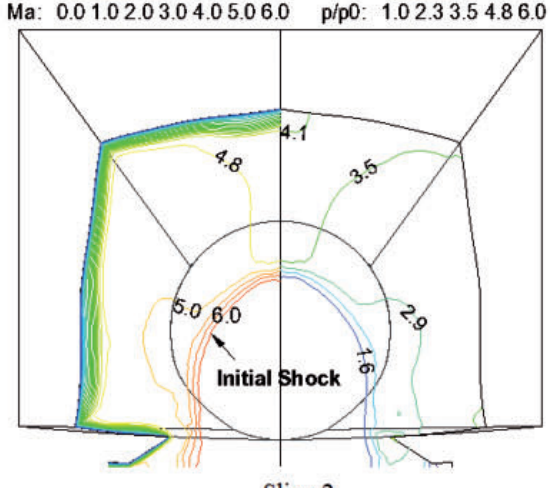

Slice 2

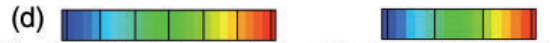

Ma: $0.01 .02 .03 .04 .05 .06 .0 \quad \mathrm{p} / \mathrm{p} 0$ : $1.0 \quad 6.0 \quad 11.016 .0$

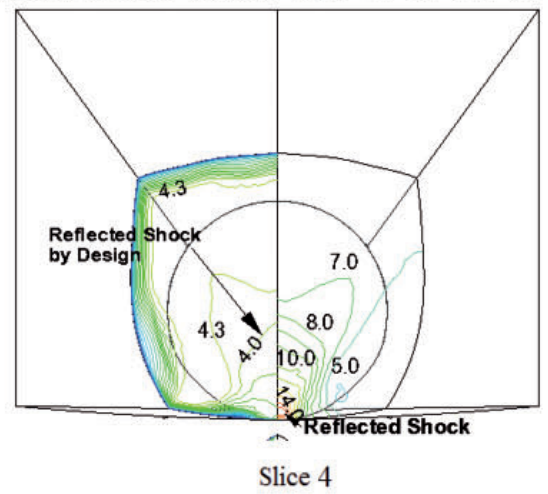

(f) \begin{tabular}{|l|l|l|l|l|l|l|l||}
\hline & & & & & & & \\
\end{tabular}

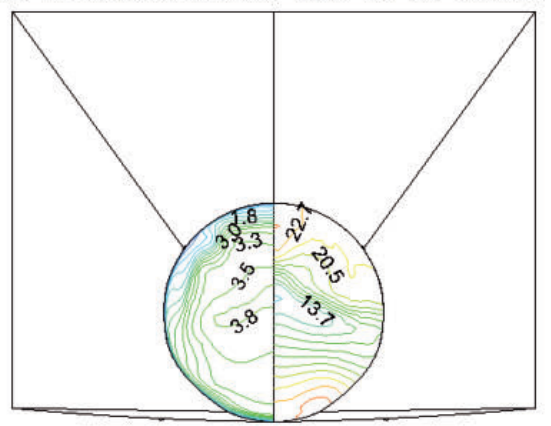

Slice 6

Figure 10. Flow field on slices of the inlet at Ma 6. 


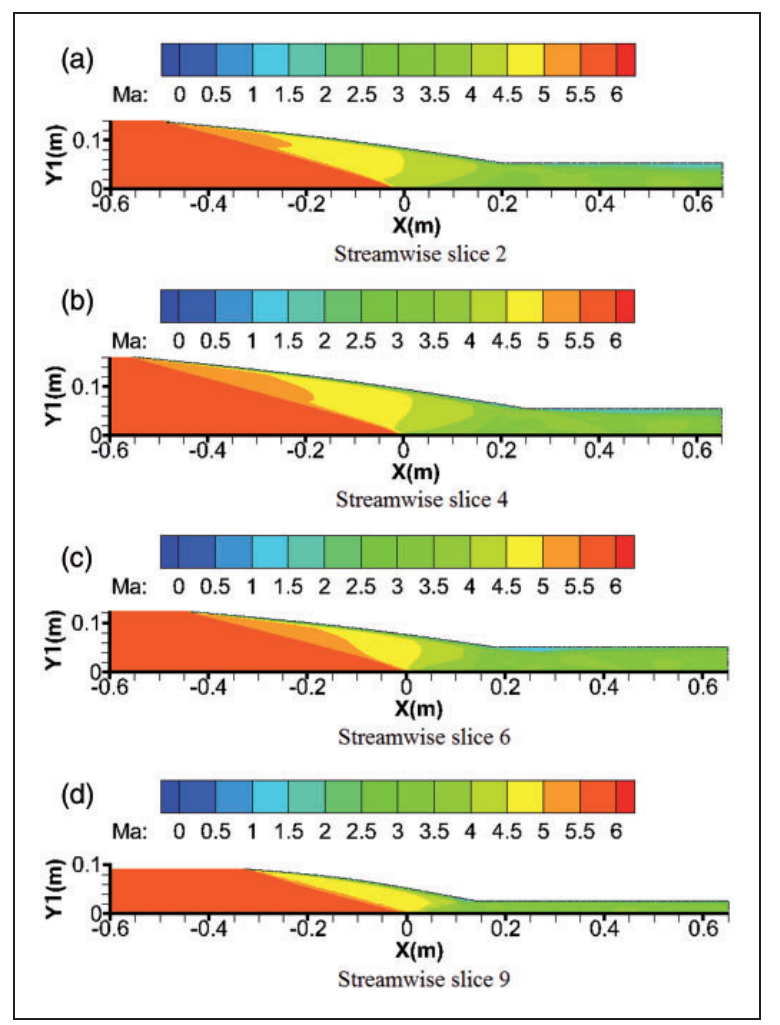

Figure II. Mach number contours on typical streamwise slices: (a) streamwise slice 2; (b) streamwise slice 4; (c) streamwise slice 6; (d) streamwise slice 9.

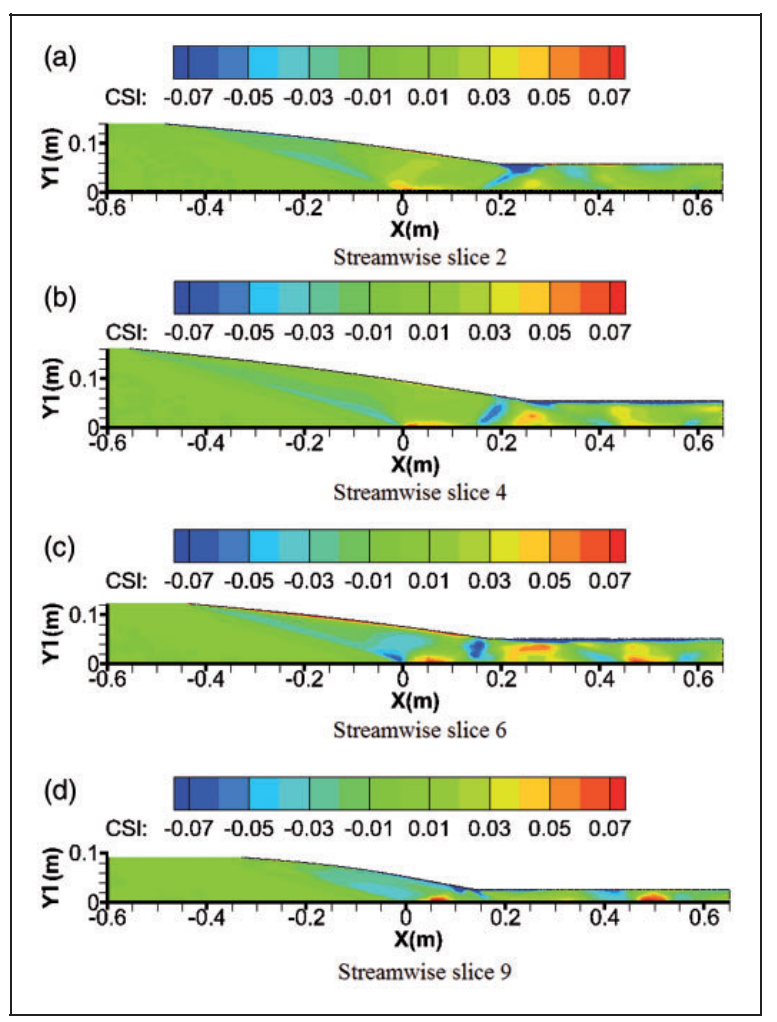

Figure 12. Contour of crossflow at typical streamwise slices, where plus denotes inward crossflow and vice versa: (a) streamwise slice 2; (b) streamwise slice 4; (c) streamwise slice 6; (d) streamwise slice 9. perfect reflection of leading edge shock is not satisfied and thus there is no free-standing reflected shock. The angle of the reflected shock at cowl lip increases with decreasing Mach number, and the shock reflection in the isolator is worse than it is at the design point. Figure 14 displays Mach number contours on the aforementioned slices. The leading edge shock remains nonaxisymmetric; however, the Mach number distribution behind the cowl reflected shock remains fairly uniform. The reflected shock does impinge on the side wall, however, neither large recirculation zone nor large zone of low Mach number is observed. The optimization achieves good control of shock strength. Although the shock reflection exists in the isolator, the Mach number is not sensitive to the weak reflection. The mainstream Mach number is quite uniform, ranging from 2 to 2.2. Generally, the flow field at off-design points exhibits fairly good flow behaviors as well.

\section{Performances of the inlet}

The inlet performances are listed in Table 1. At the design point of $M a 6$, the mass capture of the inlet attains $99.5 \%$, and the cowl lip ride nearly on the leading edge shock, where the mass loss of $0.5 \%$ arises from the backward cutting of the cowl lip. The mass flow averaged Mach number is 3.055 at the exit of isolator, while the mass flow weighed total pressure recovery reaches $59.36 \%$.

At the takeover point of $M a$ 4, the mass capture ratio remains as high as $87.23 \%$ and the inlet has a total pressure recovery coefficient of $79.46 \%$. The high mass capture at the takeover point endows the engine a high thrust and provides a necessary condition for self-reliant acceleration.

Referring to the evaluation method in Smart, ${ }^{1}$ Figure 15 shows a comparison of current constant contraction inlet with three-dimensional inlets in You et al., ${ }^{20}$ Smart, ${ }^{21}$ and Billig. ${ }^{22}$ The mass flow weighed total pressure recovery is plotted versus Mach number. Taking into account the difference in contraction ratio, the constant contraction inlet designed using the current methodology promises superior viscous performance, which is believed to be a result of both the constant contraction discretization of the flow field and the full NS CFD optimization of each steam tube in the design procedure.

\section{Conclusions}

The present paper proposes a design method for hypersonic inlets with shape transition to achieve high compression efficiency while keeping flow uniformity as much as possible. And it can be applied to an arbitrary inlet capture.

Based on 1D aerodynamics, it is the necessary condition for the uniformity of a flow field that any 


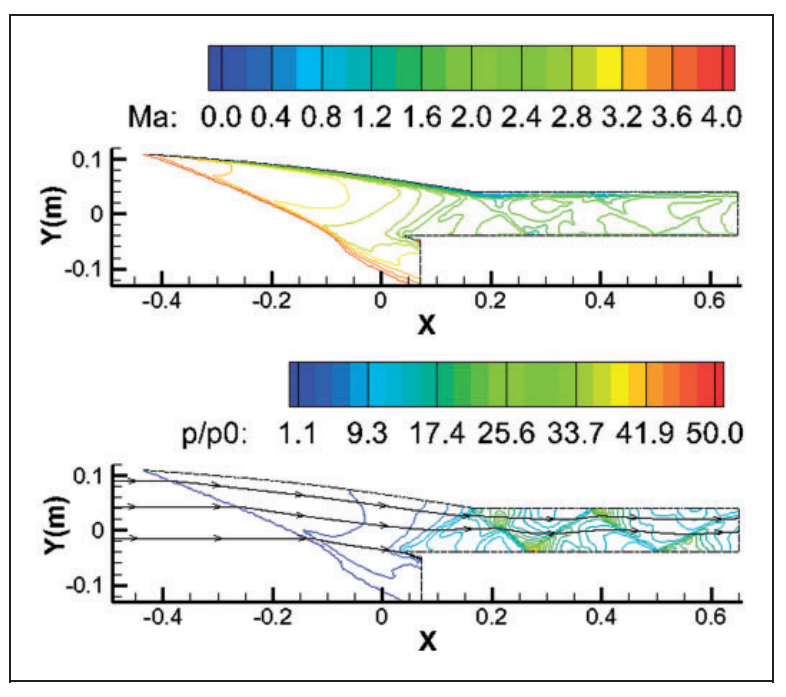

Figure 13. Flow fields on the inlet symmetric plane at Ma 4.

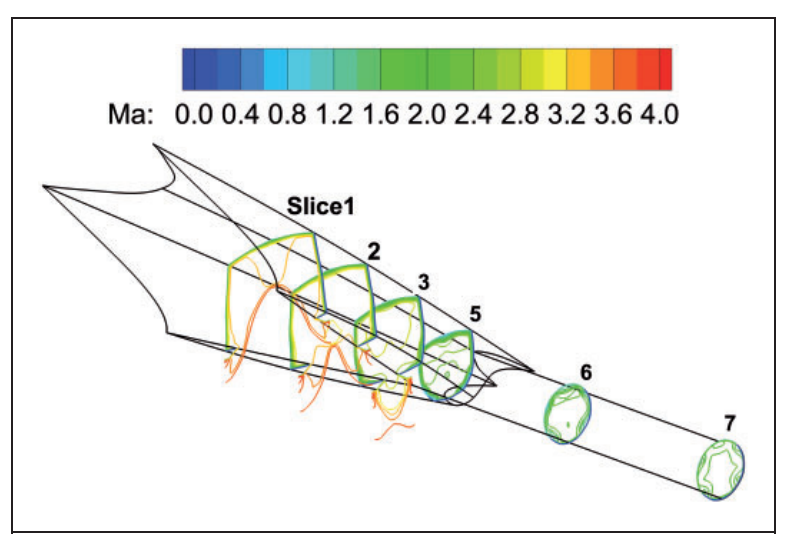

Figure 14. Pictorial views of inlet flow field at Ma 4.

Table I. Performances at the isolator exit for the design and the takeover points.

\begin{tabular}{lllll}
\hline & $\varphi$ & $\pi_{30}$ & $\sigma_{30}$ & $M a_{3}$ \\
\hline Design point & 0.9950 & 20.77 & 0.5936 & 3.055 \\
Takeover point & 0.8723 & 17.00 & 0.7946 & 1.930 \\
\hline
\end{tabular}

streamtube having the same contraction ratio. For inward turning inlets with curved leading edge shock, we propose a weakened version of the "constant contraction" condition, i.e. the "constant contraction of discrete streamtubes". According to this condition, we divide the 3D flow field into a series of discrete axisymmetric streamtubes with constant contraction ratio and continuously varying RDP, each of which is optimized via $C F D$ to acquire high compression efficiency. We first control their length-to-height ratio to endow them similar flow behaviors and then properly match their shock reflection positions so that the crosswise pressure gradient is well controlled and each streamtube keeps the desired flow pattern as much as possible.

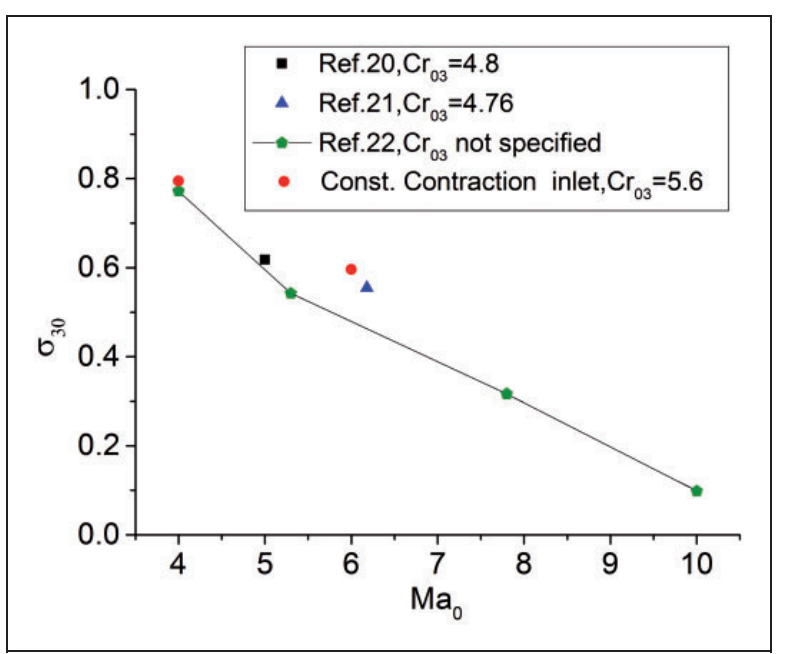

Figure 15. Comparison of three-dimensional hypersonic inlet performance.

To validate the present method, we design a rectangular-to-circular inlet with the design point of $M a 6$. Numerical simulations show that the inlet well maintains the designed flow characteristics and displays high performances at design point with relatively uniform Mach number distribution. The leading edge shock converges onto a straight vertical shock reflection line, while the cowl lip approximately rides the leading edge shock with a mass capture of $99.5 \%$. The matching and assembling of discrete streamtubes well controls the crossflow between adjacent streamtubes. On typical stream surfaces, the crossflow intensity is generally below $3 \%$ so that the desired inlet flow pattern can be achieved. The optimized design endows the inlet a high compression efficiency, to be specific, a total pressure recovery coefficient of $59.36 \%$ at static pressure ratio of 20.77. Examination of the off-design performance down to $M a 4.0$ indicates a mass capture of $87.23 \%$ with no large-scale separation. At the exit of isolator, the pressure ratio and total pressure recovery coefficient attain 17.00 and $79.46 \%$, respectively, and the flow is fairly uniform there.

These results demonstrate that the present methodology based on constant contraction of discrete streamtubes provides an effective tool for the design of high efficiency shape transition inlets.

\section{Conflict of interest}

The authors declared no potential conflicts of interest with respect to the research, authorship, and/or publication of this article.

\section{Funding}

This work was supported by the National Natural Science Foundation of China (Grant No.91216115 and 11472279).

\section{Acknowledgments}

The authors wish to thank all the members of our team. The authors will be appreciated for the Department of 
Aerospace Technology, National University of Defense Technology of China for supporting this work.

\section{References}

1. Smart MK. Design of three-dimensional hypersonic inlets with rectangular-to-elliptical shape transition. J Propul Power 1999; 15: 408-416.

2. Smart MK and White JA. Computational investigation of the performance and back-pressure limits of a hypersonic inlet. In: 40th AIAA aerospace sciences meeting and exhibit, Nevada, USA, 14-17 January 2003. AIAA 2002-0508..

3. Gollan RJ and Smart MK. Design of modular shapetransition inlets for a conical hypersonic vehicle. J Propul Power 2013; 29: 832-838.

4. Barger RL. A procedure for designing forebodies with constraints on cross-section shape and axial area distribution. NASA TP-1881, 1981.

5. Molder S and Szpiro EJ. Busemann inlet for hypersonic speeds. J Spacecraft Rockets 1966; 3: 1303-1304.

6. Sun B, Zhang K, Wang C, et al. Investigation on a streamtraced hypersonic Busemann inlet. Proc IMechE, Part G: J Aerospace Engineering 2010; 224: 57-63.

7. Taylor T and Van Wie DM. Performance analysis of hypersonic shape-changing inlets derived from morphing streamline traced flowpaths. In: 15th AIAA international space planes and hypersonic systems and technologies conference, Dayton, OH, USA, 28 April to 1 May 2008. AIAA-2008-2635.

8. Nan, X. and Zhang, K. Numerical and experimental investigation on hypersonic inward turning inlets with basic flowfield using arc tangent curve law of pressure rise. In: 17th AIAA international space planes and hypersonic systems and technologies conference, San Francisco, CA, USA, 11-14 April 2011. AIAA 20112270.

9. You Y and Liang D. Design concept of three dimensional section controllable internal wave rider hypersonic inlet. Sci China Ser E 2009; 52: 2017-2028.

10. Sobieczky H, Dougherty FC and Jones K. Hypersonic waverider design from given shock waves. In: 1st international waverider symposium, University of Maryland, USA, 17-19 October 1990.

11. Sabean JW and Lewis MJ. Computational optimization of a hypersonic rectangular-to- circular inlet. J Propul Power 2001; 17: 571-578.

12. Burgreen $\mathrm{G}$, Baysal $\mathrm{O}$ and Eleshaky M. Improving the efficiency of aerodynamic shape optimization. AIAA J 1994; 32: 69-76.

13. Billig FS and Kothari AP. Streamline tracing: Techniques for designing hypersonic vehicles. J Propul Power 2000; 16: 465-471.

14. Xiao Y, Yue L and Gong P. Investigation on a truncated stream-line traced hypersonic Busemann inlet. In: 15th AIAA international space planes and hypersonic systems and technologies conference, Dayton, $\mathrm{OH}$, USA, 28 April to 1 May 2008. AIAA 2008-2634.

15. Matthews AJ and Jones TV. Design and test of a modular waverider hypersonic intake. J Propul Power 2006; 22: 913-920.

16. Abramson MA, Audet C and Dennis Jr JE. Analysis of generalized pattern searches. SIAM J Optim 2003; 13: 889-903.

17. Conn AR, Gould NIM and Toint PL. A Globally convergent augmented Lagrangian barrier algorithm for optimization with general inequality constraints and simple bounds. Math Comput 1997; 66: 261-288.

18. Abramson MA. Pattern search filter algorithms for mixed variable general constrained optimization problems. PhD Thesis, Rice University, USA, August 2002.

19. Lu H, Yue L, Xiao Y, et al. Interaction of isentropic compression waves with a bow shock. AIAA J 2013; 51: 2474-2484.

20. You Y, Liang D and Cai K. High enthalpy wind tunnel tests of three-dimensional section controllable internal waverider hypersonic inlet. In: 47th AIAA aerospace sciences meeting including the new horizons forum and aerospace exposition, Orlando, FL, USA, 5-8 January 2009. AIAA 2009-31.

21. Smart MK. Experimental testing of a hypersonic inlet with rectangular-to-elliptical shape transition. J Propul Power 2001; 22: 276-283.

22. Billig FS. Supersonic combustion ramjet missile. J Propul Power 1995; 11: 1139-1146.

\section{Appendix}

\section{Notation}

A area

$\mathrm{Cr}_{03}$ geometrical contraction ratio, $A_{0} / A_{3}$

$C p \quad$ heat capacity at constant pressure

CSI cross-section flow intensity

$p \quad$ static pressure

$P t \quad$ total pressure

$R D P \quad$ radial deviation parameter

$s \quad$ entropy

u velocity

$\gamma \quad$ heat capacity ratio

$\varphi \quad$ mass capture ratio

$\pi_{30} \quad$ outlet-inlet pressure ratio

$\sigma_{30} \quad$ outlet-inlet total pressure recovery

\section{Subscripts}

0

3 at the entrance of the inlet at the exit of the isolator 